\title{
Long-term effects of traumatic stress on subsequent contextual fear conditioning in rats
}

Rie Ryoke ${ }^{\mathrm{a}}$, Kazuo Yamada ${ }^{\mathrm{a} *}$, Yukio Ichitani $^{\mathrm{a}}$

${ }^{a}$ Department of Psychology and Behavioral Neuroscience, University of Tsukuba, Tsukuba, Ibaraki 305-8577, Japan

Number of Pages: 22

Number of Figures: 4

Number of Tables: 0

\section{Correspondence to:}

*K. Yamada, Ph.D.

Department of Psychology and Behavioral Neuroscience

University of Tsukuba, Tsukuba, Ibaraki 305-8577, Japan

Telefax: +81-29-853-2961

E-mail: kayamada@human.tsukuba.ac.jp

Running head: Long-lasting effects of stress on fear conditioning

Keywords: Contextual fear conditioning, open field behavior, corticosterone, metyrapone, post-traumatic stress disorder, contextual reminder, rat 


\begin{abstract}
Exposure to stressful events affects subsequent sensitivity to fear. We investigated the long-term effects of a traumatic experience on subsequent contextual fear conditioning and anxiety-like behaviors in rats (Experiment 1). In addition, we tested whether the administration of the glucocorticoid synthesis inhibitor metyrapone (MET) attenuated the sensitization of fear induced by traumatic stress (Experiment 2). Male rats were subjected to a multiple stress (MS) session, which consisted of 4 foot shocks (1 mA, $1 \mathrm{~s})$ and forced swimming for $20 \mathrm{~min}$, followed by exposure to a situational reminder 7 days after the MS session. MET ( 25 or 100 $\mathrm{mg} / \mathrm{kg}$, intraperitoneal) was administered $30 \mathrm{~min}$ before MS. The contextual fear conditioning was performed 14 days after MS. MS enhanced the conditioned fear response for at least 14 days after the conditioning, and pretreatment with MET did not affect the enhancement of conditioned fear. These results suggest that glucocorticoid secretion triggered by MS is not involved in regulating the long-term stress-induced sensitization of fear.
\end{abstract}




\section{Introduction}

It is well recognized that stressful or emotionally arousing experiences enhance learning and memory. These reactions represent adaptive phenomena aimed at increasing the ability to avoid life threat that have been previously encountered. However, the memory of a danger may lead to long-lasting behavioral changes. Individuals who have faced traumatic events can often vividly retrieve the traumatic experiences to the extent that fright and anxiety are sustained. For example, posttraumatic stress disorder (PTSD) is an anxiety disorder that may develop after exposure to a strongly traumatic event.

Stress, fear, and anxiety are associated with learning and memory processes in animals [1]. For example, Pavlovian fear conditioning is a behavioral paradigm typically used to evaluate the strength of aversive memory in rodents [2] and it has been reported that previous exposure to a stressful treatment enhances subsequent fear conditioning [3-5]. This phenomenon may correspond to the hyper-sensitized reaction observed in patients with PTSD, in whom the magnitude of the response is more appropriate to the original traumatizing event than it is to current conditions [6].

Long-term effects of exposure to a single stressor have been reported previously and, the search for putative animal models of PTSD has focused on these long-term consequences [7]. A few previous studies have reported that a single session of stress can have long-lasting effects, including development of anxiety-like behaviors and neurobiological changes. For example, a social stress was able to induce long-lasting behavioral changes suggestive of enhanced anxiety and depression-like symptoms [8]. In a different study, ketoconazole, an inhibitor of steroid synthesis, prevented the long-lasting effects of predator stress on an anxiety-like behavior [9]. Pynoos et al. [10], reported that, after a severe shock, mice exposed to a situational reminder (without shocks) showed enhanced startle reflexes in an acoustic startle response test. This 
experimental paradigm was based on the finding that, with the exception of repeated trauma, traumatized individuals are often confronted with reminders of the traumatic event, but not with the traumatic event itself. The authors suggested that situational reminders of the traumatic event contributed to the reactivity in, and chronic aspects of, PTSD [10].

The biological mechanisms responsible for the stress-induced enhancement of conditioned fear have not yet been elucidated. Interestingly, Rau et al. [4] demonstrated that the stress-induced enhancement of conditioned fear was not simply the summation or generalization of fear, because pre-stress administration of an $N$-methyl-D-aspartate (NMDA) receptor antagonist (which generally prevents memory consolidation and retrieval) did not prevent stress-enhanced fear learning. Therefore, it was suggested that enhanced fear memory might not depend on associative memory of the traumatic event.

Corticosterone (CORT) is a glucocorticoid that has complex effects on cognitive and emotional functions. For example, research in learning and memory has reported that CORT has multifaceted actions on memory processes, such as enhancing the acquisition of new information and impairing the retrieval of memory [11]. In addition, many studies have indicated that administration of CORT can facilitate fear conditioning, while a pre- and post-training injection of either a CORT synthesis inhibitor or a glucocorticoid receptor antagonist impaired fear conditioning [3,12]. Multiple studies have demonstrated that administration of either a glucocorticoid synthesis inhibitor or a glucocorticoid receptor antagonist before exposure to restraint stress prevented the anxiogenic effects of the stressor $[13,14]$.

It is well established that exposure to stress activates the hypothalamus-pituitary-adrenal (HPA) axis, which results in CORT secretion. Therefore, the first aim of the present study was to 
examine whether a multiple stressor (MS), consisting of foot shocks and forced swimming, had long-term effects on anxiety-like behavior and contextual fear conditioning. Compared to just foot shocks or forced swimming, MS strongly affected subsequent conditioned fear in our preliminary experiments. The hypothesis was that the stress-induced secretion of CORT during a traumatic event contributes to the post-trauma enhancement of conditioned fear. An additional aim was to investigate whether a situational reminder of the traumatic event enhanced the effects of the MS. A final goal was to investigate whether pre-MS treatment with metyrapone (MET), a glucocorticoid synthesis inhibitor, affected the MS-induced enhancement of conditioned fear. The current results suggest that glucocorticoid secretion, triggered by MS, is not involved in regulating long-lasting stress-induced behavioral changes.

\section{Material and methods}

\subsection{Animals}

Adult male Wistar-Imamichi rats (age range 8-12 weeks) were purchased from the Institute for Animal Reproduction (Ibaraki, Japan). Rats were housed in individual cages and maintained on a $12 \mathrm{~h} / 12 \mathrm{~h}$ light/dark cycle at a controlled ambient temperature $\left(23 \pm 1^{\circ} \mathrm{C}\right)$. All experiments were carried out according to the guidelines for the Care and Use of Animals approved by the University of Tsukuba Committee on Animal Research.

\subsection{Drugs}

The glucocorticoid synthesis inhibitor, 2-methyl-1,2-di-3-pyridyl-1-propanone (metyrapone (MET); Sigma-Aldrich, St. Louis, MO USA), was dissolved in a solution of $45 \%$ 2-hydroxypropyl- $\beta$-cyclodextrin (HBC; Sigma-Aldrich). MET inhibits enzymatic conversion, by 11-beta-hydroxylase, of deoxycorticosterone to CORT, thus inhibiting CORT synthesis and subsequent release into the bloodstream. MET (dose: 25 or $100 \mathrm{mg} / \mathrm{kg}$, volume: $1 \mathrm{~mL} / \mathrm{kg}$, IP) was injected 30 min before the rat was placed in a foot-shock chamber, used to deliver MS. The 
MET doses were chosen based on previous studies performed in rats exhibiting effects of fear conditioning and anxiety-like behavior $[15,16]$. Control rats received a similar volume of $45 \%$ HBC solution.

\subsection{Apparatuses}

\subsubsection{Multiple stress}

In this study, 2 different shock chambers were used to deliver the electric foot shocks (Context A: $30 \times 25 \times 30 \mathrm{~cm}$, O'Hara \& Co., Ltd., Tokyo, Japan) and to conduct the fear conditioning (Context B). These chambers differed in their context, including the illumination, background, and sound. Electric shocks (used as the MS) were delivered in Context A, which was positioned inside a sound-attenuating chamber $(100 \times 45 \times 60 \mathrm{~cm}$, Tech Serv. Inc., MD). The sidewalls consisted of opaque black acrylic (Plexiglas, Dow Chemical, Philadelphia, USA) and the back and the front walls consisted of clear acrylic (Plexiglas, Dow Chemical) attached to a sheet of paper with a black stripe. The lid consisted of clear acrylic placed below an incandescent bulb. For the delivery of scrambled shocks, the floor consisted of 19 steel rods (diameter: $5 \mathrm{~mm}$ ) spaced $1.5 \mathrm{~cm}$ apart and wired to a shock generator (O’Hara \& Co., Ltd.). Forced swimming was performed in an opaque-blue plastic bucket (height: $50 \mathrm{~cm}$, diameter: $40 \mathrm{~cm}$ ). The bucket was filled with water to a depth of $30 \mathrm{~cm}$.

\subsubsection{Open field}

The locomotor behavior of rats was videotaped in an open field test (OFT) chamber. The chamber was an open-top box $(90 \times 90 \times 45 \mathrm{~cm})$ that consisted of black polyvinylchloride walls and a gray floor (O'Hara \& Co., Ltd.). The box was illuminated by 4 incandescent bulbs installed on the ceiling. The brightness of the center of the floor was $52.51 \mathrm{x}$. A video camera was placed above the chamber.

\subsubsection{Fear conditioning}


An automated computer-controlled system (O’Hara \& Co., Ltd.) was used in the habituation, conditioning, and retention test phases. The fear-conditioning chamber (Context B: $25 \times 20 \times 30$ $\mathrm{cm})$ was constructed of clear acrylic walls and included a lid with a hole in the center (O'Hara \& Co., Ltd.). For delivering electric shocks, the Context B chamber was equipped with a grid floor made of 16 stainless-steel rods (diameter: $5 \mathrm{~mm}, 10 \mathrm{~mm}$ apart). The chamber was located within a sound-attenuating box $(70 \times 60 \times 60 \mathrm{~cm}$; Muromachi Kikai Co., Ltd., Tokyo, Japan) with white inside walls and a ventilation fan that provided fresh air, background noise (50 dB), and illumination (200 lx).

\subsection{Procedure}

\subsubsection{Experiment 1}

\subsubsection{Multiple stress (MS)}

Rats were randomly assigned to either a MS group or non-stressed (NMS) group . Rats were gently transported to the behavioral experimentation room in a stainless-steel black box (transportation box, $20 \times 40 \times 20 \mathrm{~cm}$ ) that consisted of opaque black walls and lid and an opaque-black smooth polypropylene floor. Rats were left in the transportation box for $20 \mathrm{~min}$ after transportation, prior to placement in the respective testing apparatuses. Rats in the MS group were first placed into the Context A chamber. They were left in that for approximately 4 min, and then received 4 electric foot shocks (1 mA, duration: $1 \mathrm{~s})$ with an inter-shock interval that varied from 4-6 min (Total time in Context A: $25 \mathrm{~min}$ ). The rats in the MS group were then placed in the forced swimming chamber and were subjected to forced swimming for $20 \mathrm{~min}$ immediately after footshocks. Rats in the NMS group were merely exposed to the Context A chamber, but did not receive the shocks (Total time in Context A: 25 min). Following Context A, NMS rats were then placed into a plastic cage with new bedding (Waiting period, duration: 20 min). After either forced swimming (MS group) or the waiting period (NMS group), all animals were returned to their room in the transportation box. The procedure for this experiment is 
summarized in Figure 1.

\subsubsection{Situational reminder}

Seven days after MS, half of the rats in MS group were exposed to a situational reminder (MS+R group) by placing them in the transportation box for 3 min. Finally, 31 animals were randomly divided into three groups: NMS $(n=9), \operatorname{MS}-\mathrm{R}(\mathrm{n}=11), \operatorname{MS}+\mathrm{R}(\mathrm{n}=11)$.

\subsubsection{Open field test and contextual fear conditioning}

The OFT was performed the day after MS and 23 days after the MS session. On the day of OFT testing, each rat was transported in their home cage from the colony room to the OFT room. At the start of testing, each rat was placed in the left corner of the arena and its behavior was recorded for $10 \mathrm{~min}$. The total distance and time spent in the central and peripheral areas were recorded using an automated image analysis software (ImageJ, National Institutes of Health, Bethesda, MD USA). Other variables, including the number of rearings, grooming, and instances of leaning against the wall, were counted via careful monitoring during the observation time. At the end of the observation period, the defecation boli were also counted.

Contextual fear conditioning consisted of habituation, conditioning, and 3 retention tests. Each rat was transported in the home cage from the colony room to the fear-conditioning room. In the habituation phase, rats were exposed to the Context B chamber for 4 consecutive days, $5 \mathrm{~min} / \mathrm{d}$. In the conditioning phase (14 $\mathrm{d}$ after MS), each rat was placed into the Context B chamber and allowed to habituate for $2 \mathrm{~min}$, prior to receiving 2 mild foot shocks $(0.1 \mathrm{~mA}$, duration: $2 \mathrm{~s}$, inter-shock interval: $1 \mathrm{~min}$ ). The rat was removed from the Context B chamber immediately after the presentation of the second foot shock. In the retention test phase, each rat was again placed into the Context $\mathrm{B}$ chamber and was kept there for $3 \mathrm{~min}$, without receiving shocks. Retention testing took place on the day following the conditioning phase (Retention Test 1) and Days 8 (Retention Test 2) and 16 (Retention Test 3) after the conditioning phase. 


\subsubsection{Experiment 2}

The procedures used in Experiment 2 were the same as those used in Experiment 1, with the exception that either MET or vehicle (VEH) was administrated before MS and that the OFT was not performed. In addition, all rats were subjected to the situational reminder session and blood sampling from the tail vein was performed before and after MS treatment. Rats were randomly assigned to one of four groups ( $\mathrm{n}=8$ per group). Thirty minutes before the MS treatment, MET (Dose: 25 or $100 \mathrm{mg} / \mathrm{kg}$ ) or vehicle (VEH) was injected IP into rats of the MS group (MS+MET25, MS+MET100, and MS+VEH). For the NMS group, VEH alone was injected 30 min before exposure to the context A chamber (NMS+VEH). The procedure for this experiment is shown in Figure 1.

\subsection{Quantification of corticosterone}

Since insufficient volume of blood could be obtained from some animals, total 24 blood samples, NMS+VEH $(n=7), \operatorname{MS}+\operatorname{VEH}(n=6), \operatorname{MS}+\operatorname{MET} 25(\mathrm{n}=5), \operatorname{MS}+\operatorname{MET1} 100(\mathrm{n}=6)$, underwent the following hormonal assay. The blood collected was allowed to clot at room temperature. The serum was obtained by centrifugation at 9,500 rpm and by transferring the supernatant to a clean centrifuge tube. The serum was stored at $-80^{\circ} \mathrm{C}$ until analyzed. The procedure was carried out during the light period (1400 to 1800) for 2 days. CORT levels were measured using specific enzyme immunoassays (EIA) [17], described below. Each well (ELISA Plates 9018; Corning Life Sciences, New York, NY USA) was coated with $100 \mu \mathrm{L}$ of secondary antibody solution (anti-rabbit $\gamma$-globulin serum raised in goats, $5 \mu \mathrm{g} / 200 \mu \mathrm{L}$; Seikagaku Co., Tokyo, Japan) and incubated overnight at $4^{\circ} \mathrm{C}$. Unbound antibodies were then removed from the wells by emptying and washing 3 times using a plastic pump tube and the plates were inverted to dry. Standard CORT (Wako Pure Chemical Industries, Osaka, Japan) was diluted in the assay buffer (sodium phosphate buffer, $\mathrm{pH} 7.2$, containing bovine serum albumin at $1 \mathrm{~g} / \mathrm{L}$ ). Aliquots of the standard or sample solutions $(25 \mu \mathrm{L})$, of the antiserum solution $(100 \mu \mathrm{L})$, and of the 
labeled steroid hormones $(100 \mu \mathrm{L})$ were pipetted sequentially into each well. The rabbit anti-corticosterone serum (FKA420-E; COSMO Bio, Tokyo, Japan) was diluted 800,000-fold with assay buffer. Horseradish-peroxidase-labeled CORT (FKA419) was obtained from COSMO Bio. The plates were covered and incubated overnight at $4^{\circ} \mathrm{C}$. Unbound ligands were removed and a $150-\mu \mathrm{L}$ aliquot of the substrate solution for HRP was added to each well and incubated for another $50 \mathrm{~min}$ at $30^{\circ} \mathrm{C}$. The reaction was stopped by the addition of $50 \mu \mathrm{L}$ of $4 \mathrm{~N}-\mathrm{H}_{2} \mathrm{SO}_{4}$. The absorbance at $450 \mathrm{~nm}$ of sample and standard solutions was recorded using an automatic microplate reader (Bio-Rad model 550, Bio-Rad Laboratories, Hercules, CA USA). The results of the assay were calculated using the Microplate Manager III software (Bio-Rad).

\subsection{Statistical analyses}

All data were expressed as mean \pm SEM. All statistical analyses were performed using the Statistical Program for the Social Sciences (SPSS, IBM Corp., Armonk NY USA). Data were analyzed using two-way analysis of variance (ANOVA) or repeated-measures ANOVA. Individual comparisons were evaluated using a simple main effect test, a post-hoc Bonferroni's test or Fisher's PLSD test.

\section{Results}

\subsection{Experiment 1}

\subsubsection{Open field test}

The OFT was performed on Days 2 and 23 (after MS) for 10 min (Figure 2a). While there was no significant main effect for total distance traveled by group (NMS, MS+NR, and MS+R) on Day 2, there was a significant main effect on Day $23[\mathrm{~F}(2,28)=5.29, \mathrm{p}<0.05]$. Specifically, although the total distance traveled in each of the 3 groups (NMS, MS+NR, and MS+R) was not different on Day 2, the MS+R group exhibited a significantly lower total distance traveled on 
Day 23. In addition, post hoc testing revealed that the total distance traveled by animals in the MS+R group was significantly lower than in the NMS group $(\mathrm{p}<0.01)$.

There was a significant main effect for leaning-against-the-wall events by group (NMS, MS+NR, and MS+R) on Day $23[\mathrm{~F}(2,28)=5.62, \mathrm{p}<0.01]$ (Figure 2b). Post hoc testing revealed that the number of leaning-against-the-wall events in the MS+R group was significantly lower than that in NMS group $(\mathrm{p}<0.05)$. For Days 2 and 23 , there was no difference among the groups in terms of the time spent in the central area of the OFT arena.

\subsubsection{Contextual fear conditioning}

The level of freezing in MS+R group was consistently higher across the 3 retention tests, compared to the NMS and MS+NR groups (Figure 3). In addition, the MS+NR group exhibited a higher level of freezing than the NMS group in Test 1, but not in Tests 2 and 3. There were significant main effects of group $[\mathrm{F}(2,28)=5.4, \mathrm{p}<0.05]$ and trial $[\mathrm{F}(3,84)=30.98, \mathrm{p}<0.01]$ and a significant interaction between group and trial $[\mathrm{F}(6,84)=2.82, \mathrm{p}<0.05]$ for the 3 groups (NMS, MS+NR, and MS+R) during the contextual fear conditioning experiment. Post hoc testing showed that, in Tests 1 and 2, the MS+R group had a higher freezing percentage, than the NMS group $(\mathrm{p}<0.05)$, but not compared to the MS+NR group. In Test 3, the freezing level was significantly higher in the MS+R group compared to both the NMS and MS+NR groups (p $<0.05)$. Moreover, the MS $+\mathrm{R}$ group exhibited a significantly higher level of freezing at all testing times compared to its baseline ( $<$ 0.05). Finally, in Tests 1 and 2, the MS+NR group displayed higher freezing, compared to the baseline $(\mathrm{p}<0.05)$.

\subsection{Experiment 2}

\subsubsection{Contextual fear conditioning}

Although animals did not show clear differences between groups (NMS+VEH, MS+VEH, 
MS+MET25, and MS+MET100) on Retention Test 1 (Figure 4a), the MS-treated groups (MS+VEH, MS+MET25, and MS+MET100) showed levels of freezing that were more than double that of the NMS+VEH group on Retention Test 2. MET treatment did not affect contextual fear conditioning. There were significant main effects of group $[F(3,28)=3.14, p$ $<0.05]$ and trial $[\mathrm{F}(3,84)=69.97, \mathrm{p}<0.01]$ and an interaction between group and trial $[\mathrm{F}(9$, $84)=2.99, p<0.01]$ during the contextual fear conditioning experiment. An analysis of simple effects indicated that the group effect was significant in both Test $2[\mathrm{~F}(3,28)=6.34, \mathrm{p}<0.01]$ and Test $3[\mathrm{~F}(3,28)=3.25, \mathrm{p}<0.05]$. Post hoc testing indicated that, in Test 2 , the freezing levels of the MS+MET25 and MS+MET100 groups were significantly higher than those in the NMS+VEH group $(\mathrm{p}<0.05)$, and that the freezing levels of the MS+MET100 group were higher than those of the MS+VEH group $(\mathrm{p}<0.05)$. Moreover, in Test 3, MS-treated rats showed higher levels of freezing than the NMS+VEH group $(\mathrm{p}<0.05)$. Finally, the freezing levels of rats injected with different doses of MET (MS+MET25 and MS+MET100) were not significantly different.

\subsubsection{CORT concentration}

Serum CORT levels were significantly elevated after MS treatment (Figure 4b). The MS+MET100 group, but not the MS+MET25 group, showed lower levels of CORT after MS. There was a significant group effect $[\mathrm{F}(3,20)=7.3, \mathrm{p}<0.01]$ on the CORT level immediately after MS. Post hoc testing indicated that the MS+VEH groups exhibited higher CORT concentrations than the NMS+VEH and MS+MET100 groups $(\mathrm{p}<0.05)$. Finally, the MS+MET100 group had a significantly lower CORT level, compared to the other groups ( $<$ 0.05), which indicated a dose-dependent effect of MET on CORT secretion.

\section{Discussion}

The primary findings of this study were that a traumatic experience, consisting of foot shocks 
and forced swimming, had long-term consequences on stress-related behavioral responses in contextual fear conditioning and open field behavior. The results from Experiment 1 demonstrated that the situational reminder of the MS enhanced these stress-induced behavioral changes. In addition, rats exposed to the MS exhibited a higher level of emotional responses in an OFT performed 3 weeks after the MS treatment, but not on the same test performed the day after the MS. Experiment 2 examined the effects of MET administration (Dose: 25 or 100 $\mathrm{mg} / \mathrm{kg}$ ) on subsequent fear conditioning, when given immediately before MS. MET treatment did not affect the MS-induced enhancement of conditioned fear. Although a high dose of MET significantly reduced the post-MS serum CORT concentration, rats in the MS group exhibited higher freezing in retention tests 8-15 days after conditioning, compared to rats in the NMS group, regardless of drug treatment.

This study demonstrated that the sensitivity to fear, induced by a single session of MS, persisted long-term in rats. Because PTSD is characterized by a long-lasting symptomatology, long-term behavioral or neurochemical deficits induced by a single session of stressors are more likely related to the development of PTSD in animal models. In the current study, enhanced fear responses to contextual fear conditioning were shown in rats of the MS group, despite the fact that fear conditioning was performed 2 weeks after MS. This result supports results of other studies that investigated long-lasting effects of a single traumatic stress in fear learning. For example, abnormal behaviors such as unnecessary chamber changes were observed in an active escape/avoidance task performed 2 weeks after inescapable foot-shock treatment $[18,19]$. However, it is possible that the increase in responsiveness observed in the active escape/avoidance task was caused by the summation of fear of the inescapable foot shocks and fear of the active escape/avoidance task, since they were administered using the same apparatus. In the current study, contextual fear conditioning was performed in a different apparatus from that used to deliver MS, allowing observation of the prolonged sensitization of the fear response 
to a new aversive stimulus.

Other animal models have been used to show long-term effects on subsequent fear conditioning. Liberzon et al. [20,21] proposed a single-prolonged stress (SPS) that produced a sensitization to negative feedback in the HPA axis and alteration of hippocampal glucocorticoid receptors 7 or 14 days after SPS. The SPS animal model exhibited enhanced contextual fear conditioning 7 or 14 days after the delivery of SPS [22,23]. Rau and Fanselow [5] showed that 15 foot-shocks could cause enhancement of fear learning performed 91 days after the delivery of the foot shocks. The results of the current study are consistent with these reports. In the present study, in addition to the enhancement of fear response in the first retention test, enhanced fear responses were demonstrated in both Tests 2 and 3, performed 1 and 2 weeks after conditioning, respectively. Thus, these results demonstrate for the first time, that in animals exposed to both MS and a situational reminder, enhancement of conditioned fear was maintained for at least 2 weeks after fear conditioning.

In this study, exposure to the situational reminder was related to an increase in MS-induced abnormal behavior. Rats exposed to both MS and a situational reminder showed exaggerated response in the OFT and contextual fear conditioning tests, compared to rats only exposed to MS (no reminder). These results were consistent with the finding that a single exposure to foot shocks followed by situational reminders induced long-lasting effects on anxiety-like behavior in rodents $[10,24,25]$. Pynoos et al. [10] demonstrated that rats that received weekly situational reminders for 6 weeks showed a progressive increase in the magnitude of the startle reflex in the acoustic startle response test, as well as in aggressive behavior. These results suggest that brief exposure to the stress-related context contributes to the maintenance of long-lasting abnormal behaviors. Exposure to a stress reminder may be reminiscent of the core symptom of PTSD, involuntary re-experiencing of the trauma [26]. 
The current results showed that MS rats exhibited hypoactivity in the OFT, performed 3 weeks after MS, but not when performed immediately after MS. This result may be related to a time-dependent sensitization, which is considered a specific clinical symptom of PTSD (i.e., in the DSM-IV-TR as "With or Without delayed onset: Onset of symptoms at least 6 months after the stressor" $[20,26,27,28]$. However, it should be noted that contextual fear conditioning could cause an exaggeration of anxiety-like behavior in MS rats in the OFT conducted 3 weeks after MS. Subsequent fear conditioning had additional anxiogenic effects in the MS group, but not on rats in the NMS group, as illustrated by the smaller total distance traveled by the MS groups in the second OFT, compared to the first OFT. To determine the time-dependent sensitization effect of MS on OFT behavior, future studies should examine whether exaggerated anxiety is observed in OFT without fear conditioning in MS rats.

Results from Experiment 2 show that prevention of CORT synthesis after MS treatment did not reverse the enhancement of the conditioned fear response. Although MET significantly reduced the serum levels of CORT immediately after MS, even the high dose of MET did not influence the expression of enhanced fear conditioning. Conversely, although MS-treated rats exhibited a higher level of spontaneous freezing than the NMS group on the first day of habituation, rats given MET $(100 \mathrm{mg} / \mathrm{kg})$ prior to MS exhibited the same amount of freezing as the NMS group (data not shown). These results suggest that fear generalization was partially observed in the MS group on the first day of habituation. The generalized fear in the MS group was eliminated by the administration of MET at a high dose, suggesting that the MS-associated memory was disrupted by MET administration. Several studies have suggested that glucocorticoids may be involved in learning and memory [29-31]. Therefore, the consolidation of the fear memory of the MS itself, but not the MS-induced enhancement of the conditioned fear response, might have been modulated by CORT. In addition, although injection of MET prior to MS 
significantly inhibited MS-induced secretion of CORT, rats treated with MET $(100 \mathrm{mg} / \mathrm{kg})$ showed almost half level of CORT compared to that of control rats. Since we could not rule out the possibility that half the amount of secretion of CORT observed in MET-treated rats caused subsequent enhancement of conditioned fear, further studies using alternative manipulations which enable total inhibition of CORT secretion, such as adrenalectomy, will be needed.

Unexpectedly, the CORT level of NMS + VEH rats after MS treatment significantly increased compared to the basal levels in Experiment 2. Since NMS rats exposed to a shock chamber without delivering foot shocks, the elevation of CORT levels in NMS rats may be attribute to the exposure to the novel environment. These findings also suggest that MS-induced enhancement of conditioned fear is not dependent solely on stress-induced elevation of CORT.

As aversive events lead to physiological stress responses that include the HPA axis and the autonomic nervous system, the present study focused on CORT secretion as a key factor responsible for behavioral sensitization to traumatic stress [29]. However, MS-induced secretion of CORT was not necessary for the long-term enhancement of contextual fear conditioning. As previously noted, CORT is involved in widespread responses to stressors, but may be just as a modulator of learning and memory related to stress. It is possible that the other stress-related hormones or neurotransmitters are capable of encoding and storing traces of traumatic stress and that the MS-induced enhancement of conditioned fear will occur. It has been reported that aversive events trigger changes in physiological, neuroendocrine, and neuropeptide responses. Therefore, additional studies are needed to identify the key substance(s) responsible for enhancing the stress-induced fear response.

\section{Conclusion}


The findings of the current investigation demonstrated that exposure to MS can lead to long-term enhancement of a conditioned fear response and to a change in emotionality. Moreover, the effects of MS were potentiated by a situational reminder. Although pre-MS MET administration impaired contextual memory of the MS itself, it did not prevent the MS-induced enhancement of conditioned fear. In conclusion, hyperactivation of the HPA-axis induced by traumatic stress does not appear to be essential in subsequent long-term sensitization to mild stress.

\section{Acknowledgements}

The authors would like to thank Dr. Takefumi Kikusui for his help in performing the ELISA experiments and Dr. Constantine Pavlides for critical reading and valuable comments on the manuscript. This study was supported in part by Grants-in-Aid for Scientific Research from Japan Society for the Promotion of Science (24653209, 24530909, 24· 1833).

\section{Declaration of interest}

The authors report no conflicts of interest. The authors alone are responsible for the content and writing of the paper. 


\section{References}

[1] Roozendaal B, McEwen BS, Chattarji S. Stress, memory and the amygdala. Nat Rev Neurosci 2009;10:423-433.

[2] Fanselow MS, DeCola JP, Young SL. Mechanisms responsible for reduced contextual conditioning with massed unsignaled unconditional stimuli. J Exp Psychol Anim Behav Process 1993;19:121-137.

[3] Cordero MI, Venero C, Kruyt ND, Sandi C. Prior exposure to a single stress session facilitates subsequent contextual fear conditioning in rats: Evidence for a role of corticosterone. Horm Behav 2003;44:338-345.

[4] Rau V, DeCola JP, Fanselow MS. Stress-induced enhancement of fear learning: an animal model of posttraumatic stress disorder. Neurosci Biobehav Rev. 2005;29:1207-1223.

[5] Rau V, Fanselow MS. Exposure to a stressor produces a long lasting enhancement of fear learning in rats. Stress 2009;12:125-133.

[6] Bremner JD, Krystal JH, Southwick SM, Charney DS. Functional neuroanatomical correlates of the effects of stress on memory. J Trauma Stress 1995;8:527-553.

[7] Armario A, Escorihuela RM, Nadal R. Long-term neuroendocrine and behavioural effects of a single exposure to stress in adult animals. Neurosci Biobehav Rev 2008;32:1121-1135.

[8] Koolhaas JM, Meerlo P, De Boer SF, Strubbe JH, Bohus B. The temporal dynamics of the stress response. Neurosci Biobehav Rev 1997;21:775-782.

[9] Cohen H, Kozlovsky N, Matar MA, Kaplan Z, Zohar J. Mapping the brain pathways of traumatic memory: inactivation of protein kinase $\mathrm{M}$ zeta in different brain regions disrupts traumatic memory processes and attenuates traumatic stress responses in rats. Eur Neuropsychopharmacol 2010;20:253-271.

[10]Pynoos RS, Ritzmann RF, Steinberg AM, Goenjian A, Prisecaru I. A behavioral animal model of posttraumatic stress disorder featuring repeated exposure to situational reminders. Biol Psychiatry 1996;39:129-134. 
[11]de Quervain DJ-F, Aerni A, Schelling G, Roozendaal B. Glucocorticoids and the regulation of memory in health and disease. Front Neuroendocrinol 2009;30:358-370.

[12] Cordero MI, Kruyt ND, Merino JJ, Sandi C. Glucocorticoid involvement in memory formation in a rat model for traumatic memory. Stress 2002;5:73-79.

[13]Calvo N, Martijena ID, Molina VA, Volosin M. Metyrapone pretreatment prevents the behavioral and neurochemical sequelae induced by stress. Brain Res 1998;800:227-235.

[14]Calvo N, Volosin M. Glucocorticoid and mineralocorticoid receptors are involved in the facilitation of anxiety-like response induced by restraint. Neuroendocrinology 2001;73:261-271.

[15]Hirani K, Sharma AN, Jain NS, Ugale RR, Chopde CT. Evaluation of GABAergic neuroactive steroid 3alpha-hydroxy-5alpha-pregnane-20-one as a neurobiological substrate for the anti-anxiety effect of ethanol in rats. Psychopharmacology (Berl) 2005;180:267-278.

[16] Yang Y-L, Chao P-K, Ro L-S, Wo Y-YP, Lu K-T. Glutamate NMDA receptors within the amygdala participate in the modulatory effect of glucocorticoids on extinction of conditioned fear in rats. Neuropsychopharmacology 2007;32:1042-1051.

[17]Kikusui T, Nakamura K, Kakuma Y, Mori Y. Early weaning augments neuroendocrine stress responses in mice. Behav Brain Res 2006;175:96-103.

[18]Koba T, Kodama Y, Shimizu K, Nomura S, Sugawara M, Kobayashi Y, Ogasawara T. Persistent behavioural changes in rats following inescapable shock stress: a potential model of posttraumatic stress disorder. World J Biol Psychiatry 2001;2:34-37.

[19] Sawamura T, Shimizu K, Nibuya M, Wakizono T, Suzuki G, Tsunoda T, Takahashi Y, Nomura S. Effect of paroxetine on a model of posttraumatic stress disorder in rats. Neurosci Lett 2004;357:37-40.

[20]Liberzon I, Krstov M, Young EA. Stress-restress: effects on ACTH and fast feedback. Psychoneuroendocrinology 1997;22:443-453. 
[21]Liberzon I, López JF, Flagel SB, Vázquez DM, Young EA. Differential regulation of hippocampal glucocorticoid receptors mRNA and fast feedback: relevance to post-traumatic stress disorder. J Neuroendocrinol 1999;11:11-17.

[22] Takahashi T, Morinobu S, Iwamoto Y, Yamawaki S. Effect of paroxetine on enhanced contextual fear induced by single prolonged stress in rats. Psychopharmacology (Berl) 2006;189:165-173.

[23]Kohda K, Harada K, Kato K, Hoshino A, Motohashi J, Yamaji T, Morinobu S, Matsuoka N, Kato N. Glucocorticoid receptor activation is involved in producing abnormal phenotypes of single-prolonged stress rats: a putative post-traumatic stress disorder model. Neuroscience 2007;148:22-33.

[24]Louvart H, Maccari S, Ducrocq F, Thomas P, Darnaudéry M. Long-term behavioural alterations in female rats after a single intense footshock followed by situational reminders. Psychoneuroendocrinology 2005;30:316-324.

[25]Louvart H, Maccari S, Lesage J, Léonhardt M, Dickes-Coopman A, Darnaudéry M. Effects of a single footshock followed by situational reminders on HPA axis and behaviour in the aversive context in male and female rats. Psychoneuroendocrinology 2006;31:92-99.

[26] American Psychiatric Association. Diagnostic and Statistical Manual of Mental Disorders (4th ed., text rev.) Washington, DC: American Psychiatric Association; 2000.

[27] Yehuda R, Antelman SM. Criteria for rationally evaluating animal models of posttraumatic stress disorder. Biol Psychiatry 1993;33:479-486.

[28]Harvey BH, Naciti C, Brand L, Stein DJ. Endocrine, cognitive and hippocampal/cortical 5HT 1A/2A receptor changes evoked by a time-dependent sensitisation (TDS) stress model in rats. Brain Res 2003;983:97-107.

[29] McEwen BS. The neurobiology of stress: from serendipity to clinical relevance. Brain Res 2000;886:172-189. 
[30]Beylin AV, Shors TJ. Glucocorticoids are necessary for enhancing the acquisition of associative memories after acute stressful experience. Horm Behav 2003;43:124-131.

[31]Park CR, Campbell AM, Woodson JC, Smith TP, Fleshner M, Diamond DM. Permissive influence of stress in the expression of a U-shaped relationship between serum corticosterone levels and spatial memory errors in rats. Dose Response 2006;4:55-74. 


\section{Figure captions}

Figure 1. Experimental design. Experiment 1 investigated the effects of a multiple stress (MS) and a situational reminder on open field test (OFT) and contextual fear conditioning. Experimental 2 investigated the effects of metyrapone prior to MS on the post-trauma enhancement of conditioned fear. Baseline blood samples were collected a day before MS.

Figure 2. Effects of multiple stress (MS) on the mean \pm SEM (a) total distance traveled and (b) number of times the rats leaned against the wall recorded in the open field test (OFT). Rats were tested for 10 min on Days 2 and 23 (days after MS session). (a) The MS+R group showed reduced open field exploration only on Day 23. (b) The MS+R group showed a reduced number of leaning-against-the-wall events only on Day $23 .{ }^{*} \mathrm{p}<0.05$

Figure 3. Effects of multiple stress (MS) on contextual fear conditioning. MS-treated rats showed higher conditional freezing than did rats in the non-multiple stress (NMS) group in Test 1. The MS+R group showed enhanced freezing in both Tests 2 and 3. ${ }^{*} \mathrm{p}<0.05$. Data are shown as mean $\pm \mathrm{SEM}$.

Figure 4. (a) Effects of metyrapone (MET) on contextual fear conditioning. Multiple stress (MS)-treated rats had higher conditional freezing than did rats in the non-multiple stress (NMS) group. MET administration prior to the MS treatment did not affect the enhancement of conditional fear on subsequent fear conditioning. ${ }^{*} \mathrm{p}<0.05$ compared to NMS+VEH. \#p $<0.05$ compared to MS+VEH. (b) Effect of MET injection prior to MS on the corticosterone response to MS. Blood samples were taken 1 day before (Baseline) and immediately after MS. The increase of the serum corticosterone concentration induced by MS was significantly suppressed by pretreatment with MET (Dose $100 \mathrm{mg} / \mathrm{kg}$ ). $* \mathrm{p}<0.05$. Data are shown as mean \pm SEM. 
Fig. 1

Exp. 1

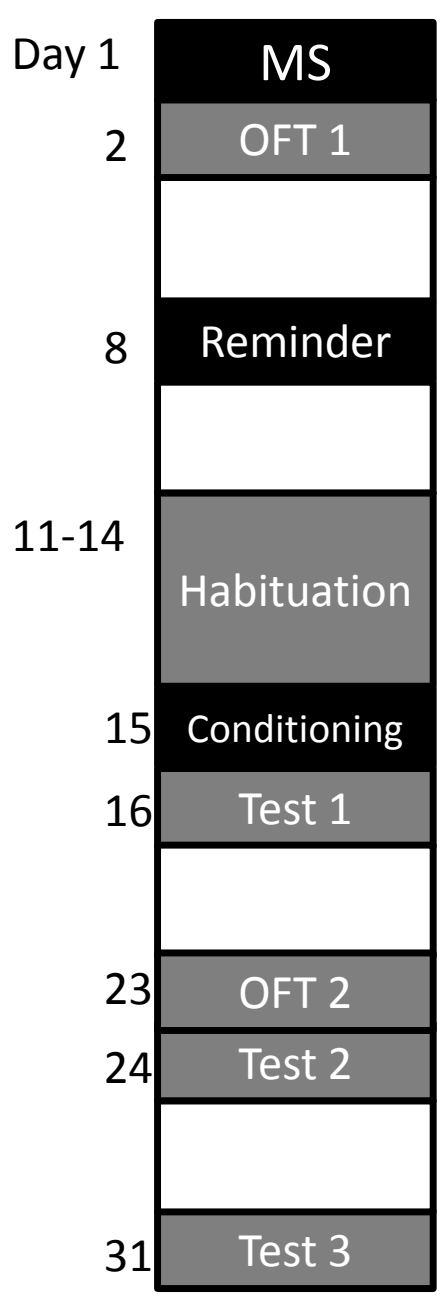

Exp. 2

metyrapone (i.p.)

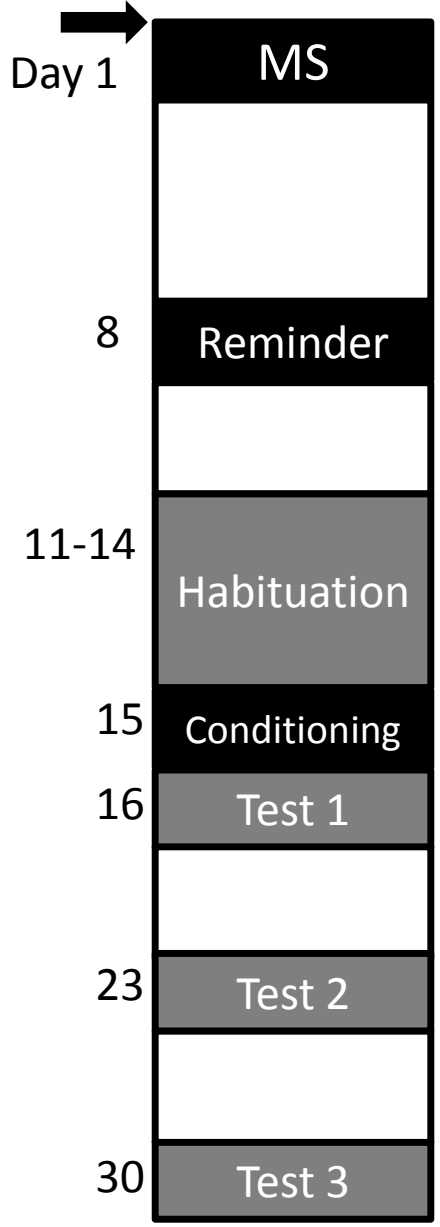



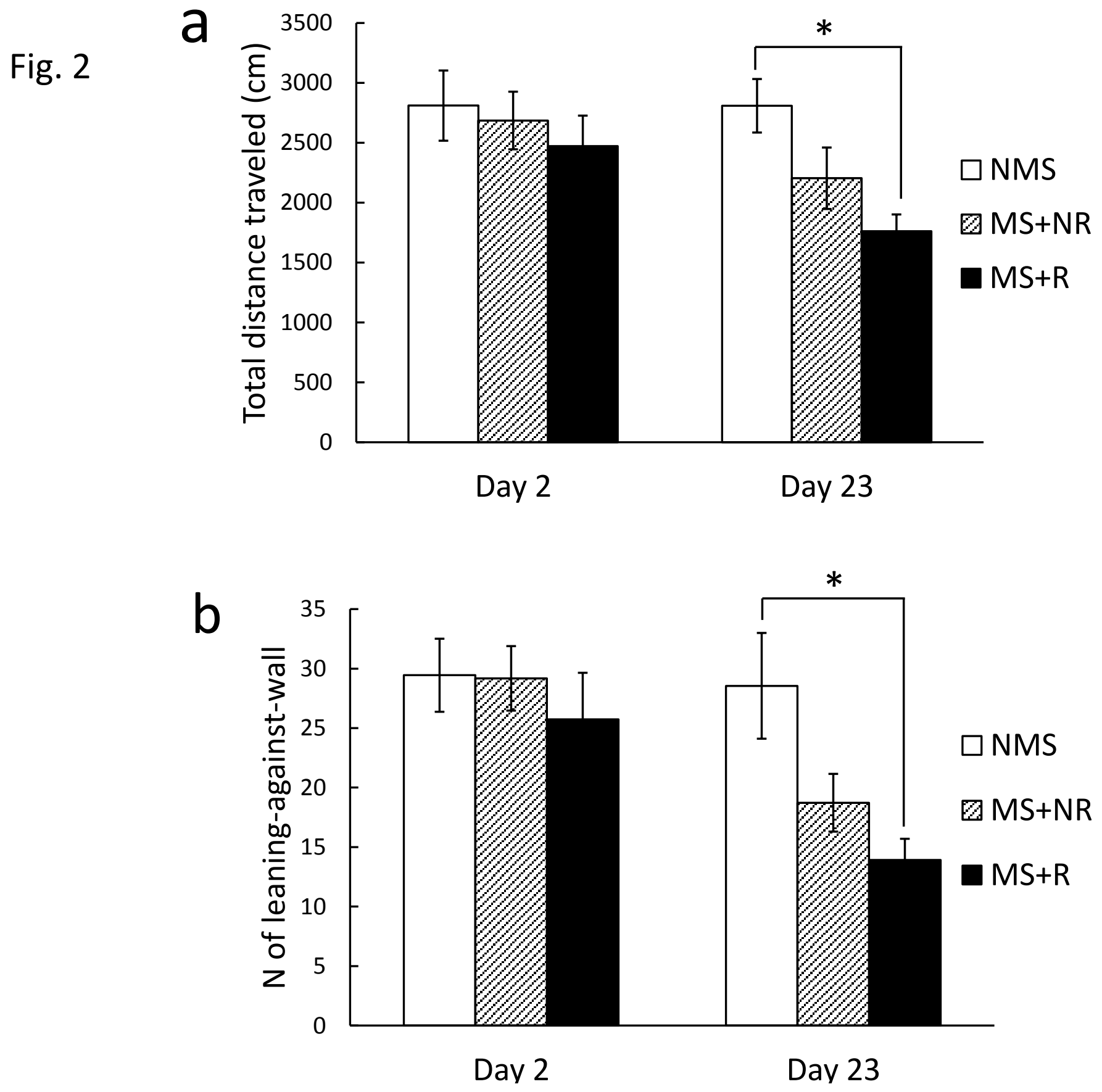
Fig. 3

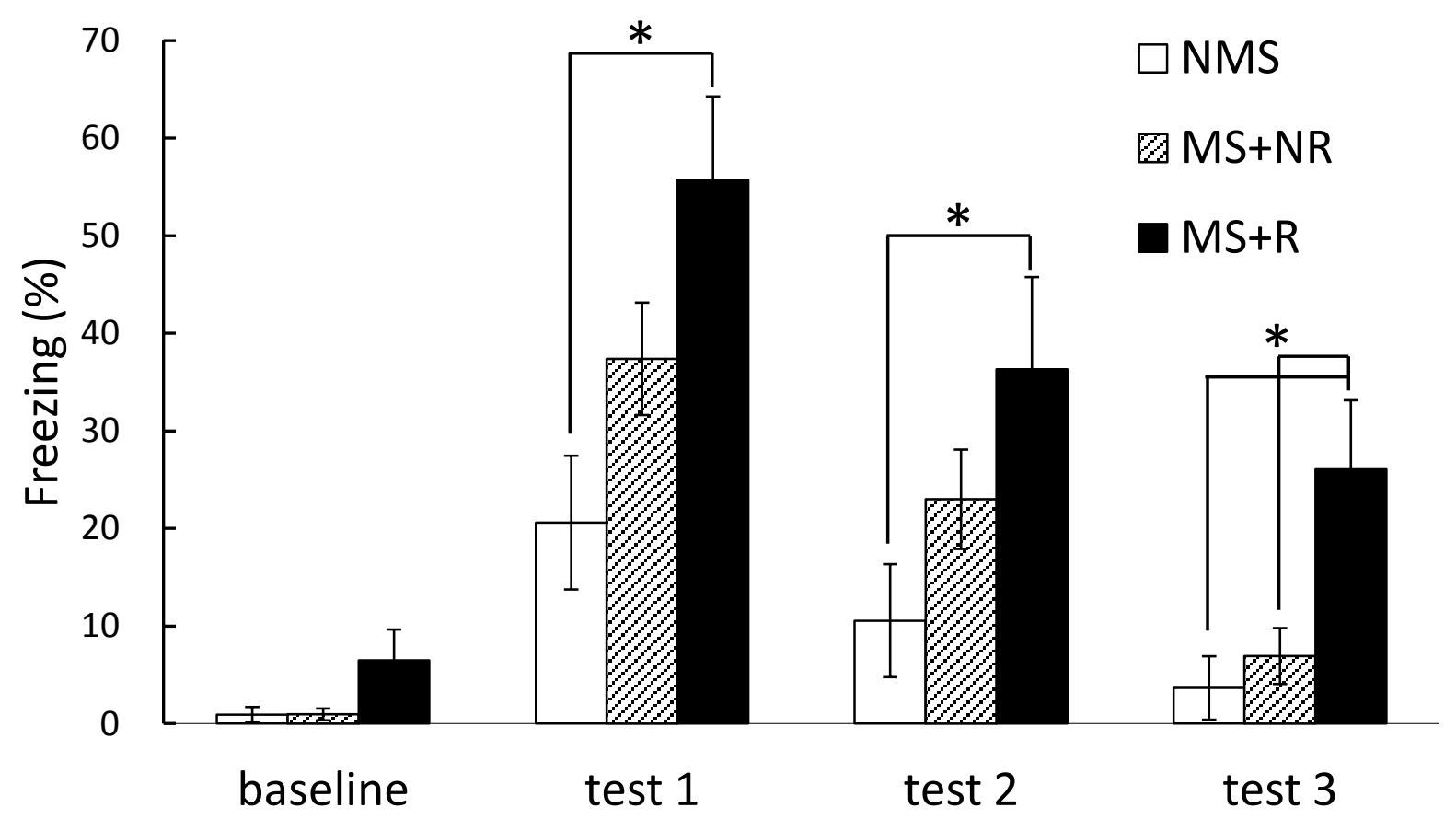


Fig. 4
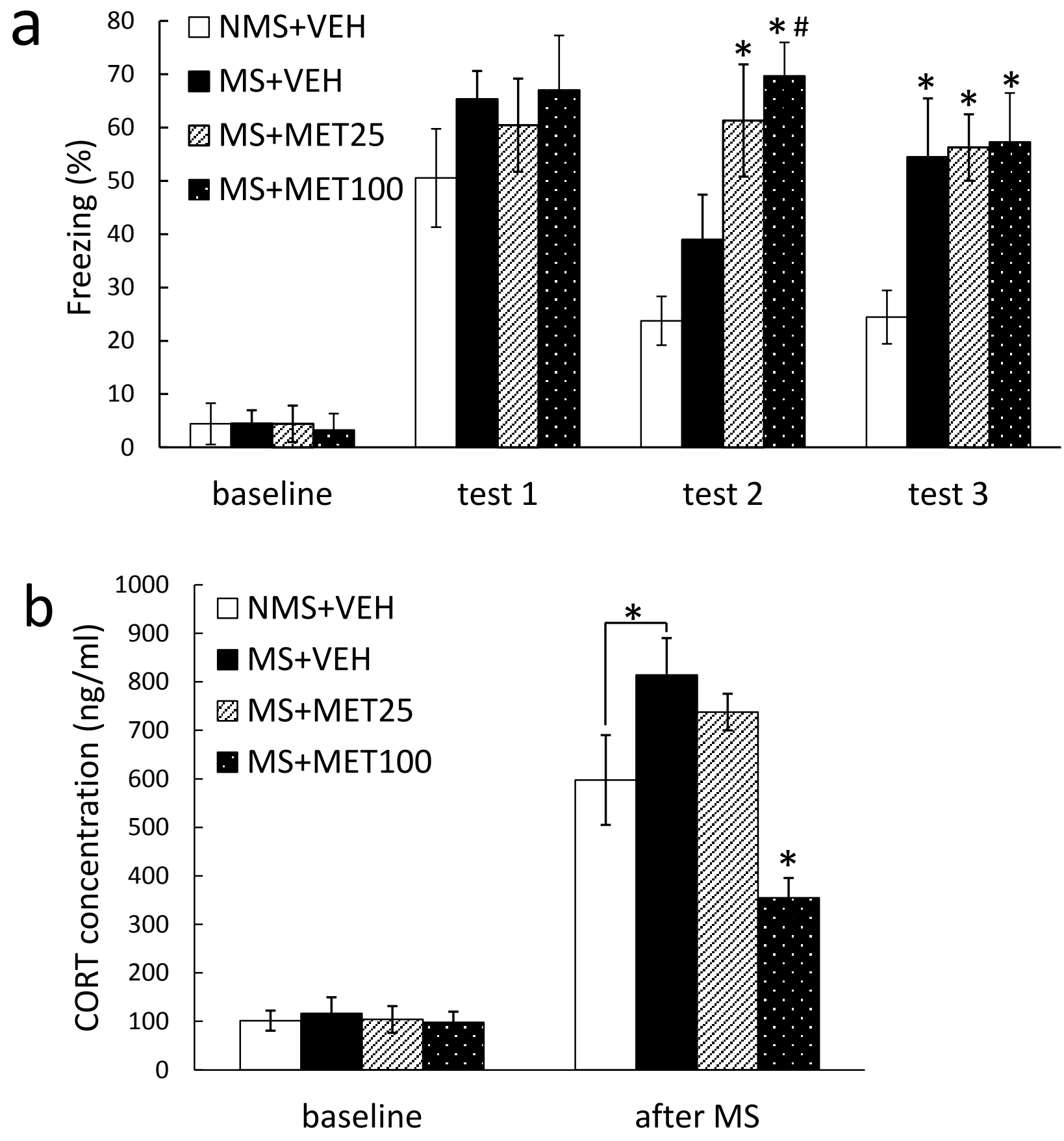